\title{
Introduction to the Minitrack Human-Computer Interaction in the Digital Economy
}

\author{
Christoph Schneider \\ IESE Business School \\ cschneider@iese.edu
}

\author{
Joseph S. Valacich \\ University of Arizona \\ valacich@arizona.edu
}

\author{
Jeffrey Jenkins \\ Brigham Young University \\ jeffrey_jenkins@,byu.edu
}

As the Human-Computer Interaction (HCI) minitrack continues to evolve we have witnessed new and exciting avenues for exploration. In 2006, this minitrack was developed to provide an outlet for a variety of HCI research streams from a variety of disciplines. In 2013, we began including the disciplines neuroscience and design science. With the ever-increasing role of information systems in all aspects of society, we moved the minitrack to the Internet and Digital Economy track in 2018, with the focus on the role of Human-Computer Interaction in the Digital Economy. Whereas traditional desktop computers continue to be important and widely used information systems - especially in organizational and home office settings - the area of human computer interaction has broadened considerably, with a proliferation of input devices, contexts, and form factors. At the same time, the design of humancomputer interfaces continues to be a crucial factor influencing users' affective and cognitive reactions and behaviors; these trends - and others - contribute to the continued need for theory-based HCI research in a variety of contexts and domains. Our aim is to get a truly cross-disciplinary understanding of HCI that informs research and impacts design practices.

The papers selected for the competitive HCI minitrack draw on this rich cross-disciplinary tradition. Given that HCI continues to evolve, we aim to provide a forum for the exchange of novel thoughts and ideas. We believe that the five papers presented in this minitrack are interesting and thought-provoking and are relevant for both research and practice. The accepted papers provide a cross-section of HCI and interface design issues in general as well as emerging contexts.

The first set of papers broadly focuses on the influence on user behaviors and intentions in different contexts. In the paper "Influencing Environmentally Sustainable Consumer Choice through Information Transparency," Dorothy Leidner, Juliana Sutanto, and Lazaros Goutas demonstrate that the sustainability of attribute information that is being disclosed will not be equally successful in terms of influencing sustainable choices. This is because the sustainability of attribute information is usually situated next to more concrete types of information (e.g. pricing information) that under certain conditions guide consumers away from the choice of sustainable products. In the second paper, titled "The Intricate Effects of Complexity and Personalization on Investment Intention in RoboAdvisory," Gregor Albrecht, Jonas Toutaoui, and Konstantin Roethke explore the interplay between website complexity and personalization within a roboadvisor's interface design and how it influences users' investment decisions. They show that personalization lowers users' intention to invest, whereas complexity has a significant positive effect on users' investment intentions and attenuates the negative impact of personalization. In the third paper, titled "Factors Influencing Usage Intentions Towards a Self-service Kiosk with Biometric Authentication," Gabriele Obermeier, Jasmin Klingersberger, and Andreas Auinger show that usage and positive word-of-mouth intentions towards a self-service kiosk with biometric authentication are strongly affected by customers' perception of convenience and relative advantage.

The second set of papers focuses on understanding user behaviors during the interaction with humancomputer interfaces. In the paper "What do Users Actually Look at During 'Zoom' Meetings? Discovery Research on Attention, Gender and Distraction Effects," Joey George, Akmal Mirsadikov, Misty Nabors, and Kent Marett explore what users actually observe during meetings using computer-based video communications tools (such as Zoom), which have become widely used during the COVID-19 pandemic. They found that while participants do pay attention to others in the meetings, gaze patterns differ between small and large groups, such that for small groups, participants look away from the screen about one-third of the time. In addition, participants often look at their own video and women look at themselves more than men. Finally, in the paper "Thinking Fast or slow? Understanding Answering Behavior Using DualProcess Theory through Mouse Cursor Movements," David Kim, Joseph Valacich, Jeffrey Jenkins, Manasvi Kumar, and Alan Dennis draw on Dual-Process Theory to explain how changes in cognitive load 
manifest as changes in mouse cursor speed. They show that participants' mouse cursor movements slow down when they are engaged in cognitively demanding tasks.

We would like to sincerely thank the researchers who contributed to this minitrack. Also, we would like to express our thanks for the outstanding efforts put forth by the many reviewers who helped ensure that the papers included in this minitrack are both interesting and relevant to the HCI field. 